\title{
Experimental investigation of liquid-liquid system drop size distribution in Taylor-Couette flow and its application in the CFD simulation
}

\author{
Reza Farzad $^{1,2^{*}}$, Stefan Puttinger ${ }^{2}$, Stefan Pirker $^{2}$ and Simon Schneiderbauer ${ }^{1,2}$ \\ ${ }^{1}$ Christian Doppler Laboratory for Multi-Scale Modeling of Multiphase Processes, Johannes Kepler University, 4040 Linz, Austria \\ ${ }^{2}$ Department of Particulate Flow Modelling, Johannes Kepler University, 4040 Linz, Austria
}

\begin{abstract}
Liquid-liquid systems are widely used in the several industries such as food, pharmaceutical, cosmetic, chemical and petroleum. Drop size distribution (DSD) plays a key role as it strongly affects the overall mass and heat transfer in the liquid-liquid systems. To understand the underlying mechanisms single drop breakup experiments have been done by several researchers in the Taylor-Couette flow; however, most of those studies concentrate on the laminar flow regime and therefore, there is no sufficient amount of data in the case of in turbulent flows. The well-defined pattern of the Taylor-Couette flow enables the possibility to investigate DSD as a function of the local fluid dynamic properties, such as shear rate, which is in contrast to more complex devices such as stirred tank reactors. This paper deals with the experimental investigation of liquid-liquid DSD in Taylor-Couette flow. From high speed camera images we found a simple correlation for the Sauter mean diameter as a function of the local shear employing image processing. It is shown that this correlation holds for different oil-in-water emulsions. Finally, this empirical correlation for the DSD is used as an input data for a CFD simulation to compute the local breakup of individual droplets in a stirred tank reactor.
\end{abstract}

\section{Introduction}

The mixture of two or more immiscible liquids, is called emulsion. Emulsions are important in several processes such as food, pharmaceutical, polymer and chemical industries. Drop size distribution (DSD) plays an essential role as it controls the overall heat and mass transfer inside the reactor [1]. Several studies are done to measure the DSD in the system. On one hand, some of these works are just focused on the single drop experiment [2-4] to define the break up kernel for the Population balance equation [5]. On the other hand, there are studies focused on the drop size distribution in the stirred tank reactor regardless of the event happens to each droplet [6,7]. The well-defined pattern of the Taylor-Couette flow enables the possibility to investigate DSD as a function of the local fluid dynamic properties, such as shear rate, which is in contrast to more complex devices such as stirred tank reactors.

Equation (1) is one of the first expressions to evaluate the Sauter mean diameter in a stirred tank reactor that was developed by the work of Shinnar and Church [8] and Chen and Middleman [6].

(1)

$$
\frac{D_{32}}{L}=A W e^{-3 / 5}
$$

Where $\mathrm{A}$ and $\mathrm{L}$ denote a constant value, which can be found experimentally and diameter of the impeller (characteristics length), respectively.

There are several models which follow the same idea as equation (1) to predict the global Sauter mean diameter[9]; however, they are not able to predict the local drop size distribution as they use global flow values such as the average dissipation rate and the average velocity in the stirred tank reactor. In this study we present an overview of the experiment of emulsion formation in a Taylor-Couette flow device to find a correlation as function of local shear rate, then implement it beside a new idea for the droplet break up in order to simulate the liquid-liquid system in a stirred tank reactor.

\section{Theory and experiment setup}

Fig.1 shows the schematic view of the experiment. The $R_{\text {in }}=112.5 \mathrm{~mm} \quad$ and $\quad R_{\text {out }}=120 \mathrm{~mm}, h=R_{\text {out }}-$ $R_{\text {in }}=7.5 \mathrm{~mm}$ is the gap between the cylinders Characteristic length. 


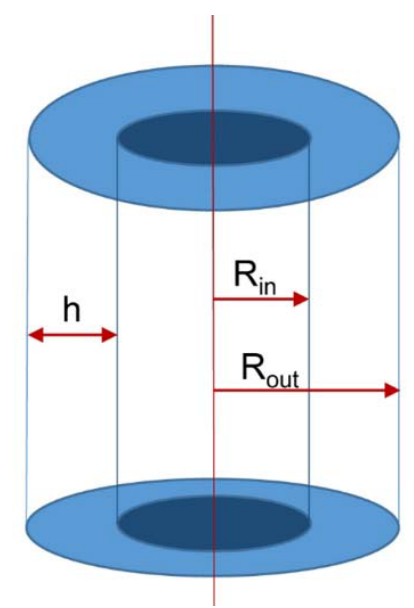

Fig. 1. Taylor-Couette flow device

shear rate in Couette flow is defined, $a=\frac{U}{h}$ where $U$ is the linear velocity of the inner cylinder. Thus, the dimensionless numbers can be defined as below:

$$
\begin{gathered}
R e=\frac{\rho_{c} a h^{2}}{\mu_{c}} \\
C a=\frac{\mu_{c} a h}{\gamma} \\
W e=\frac{\rho_{c} a^{2} h^{3}}{\gamma}
\end{gathered}
$$

Where $\mu_{c}, \rho_{c}$ and $\gamma$ denote continuous phase viscosity, continuous phase density and interfacial tension, respectively. The liquid-liquid system in this work consists of $1000 \mathrm{ml}$ of continuous phase and $10 \mathrm{ml}$ of dispersed phase. Volume fraction of the dispersed phase was kept under $1 \%$ in order to neglect the coalescence [1]. Therefore, pure break up process was considered in the current work. Table 1 shows the oils at temperature of $21{ }^{\circ} \mathrm{C}$ which were used as the dispersed phase and water was the continuous phase, respectively.

Table 1. Liquid-Liquid system properties at $21^{\circ} \mathrm{C}$

\begin{tabular}{|c|c|c|c|}
\hline & $\begin{array}{c}\text { Density } \\
\left(\mathrm{Kg} / \mathrm{m}^{3}\right)\end{array}$ & $\begin{array}{c}\text { Viscosity } \\
(\mathrm{cP})\end{array}$ & $\begin{array}{c}\text { Interfacial } \\
\text { tension } \\
(\mathrm{mN} / \mathrm{m})\end{array}$ \\
\hline Raps oil/w & 907 & 70 & 23.71 \\
\hline Soybean oil/w & 916 & 47 & 20.81 \\
\hline Silicon oil/w & 760 & 0.49 & 15.9 \\
\hline
\end{tabular}

A high speed camera (Photron FASTCAM SA2) was employed along with a $1200 \mathrm{~W}$ photography lamp to capture the movie from the droplets. ImageJ (Noncommercial scientific program) was used for post processing. Diameter was calculated based on the area of the droplets $\left(D=\sqrt{\frac{4 A}{\pi}}\right)$.

\section{Experimental investigation}

Experiment was started by the lowest possible rotational speed and it was increased gradually till maximum speed can be reach by the electromotor. Movie was captured on the specific rotational speeds for each oil.

\subsection{Results and discussion}

Fig. 2 shows the log-normal cumulative drop volume distribution of raps oil.

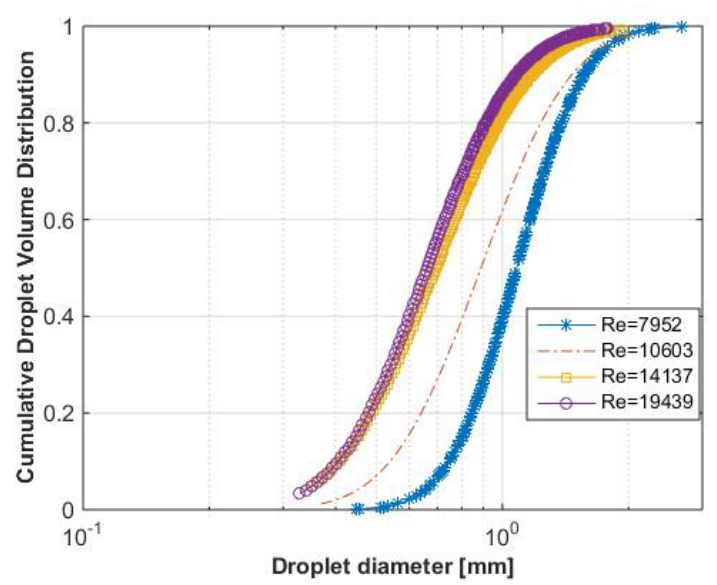

Fig. 2. Raps oil- lognormal cumulative drop volume distribution

As it can be seen in Fig.2, drop size distribution moves towards to the smaller sizes as the rotational speed $(\mathrm{Re})$ increases. It is the same trend for the other oils. We observed that the fluid properties such as interfacial tension and viscosity of the dispersed phase are important to keep the interface stable against the disruptive forces which mainly comes from dynamic pressure of the continuous phase.

Fig.3 illustrates the dimensionless Sauter mean diameter of the raps oil against shear rate for all cases. Sauter mean diameter reduces as the shear rate increases, however the trend is not linear. In particular, as the shear rate increases to very high values, it appears that the Sauter mean diameter approaches a constant value. 


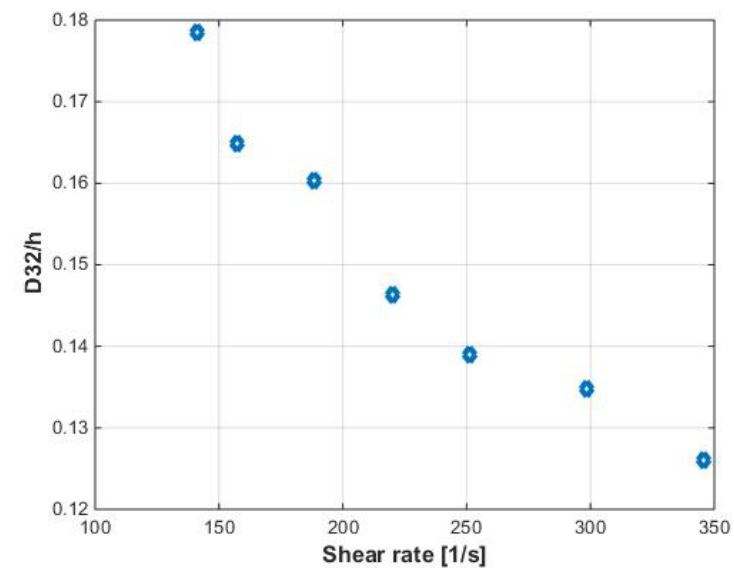

Fig. 3. Raps oil dimensionless Sauter mean diameter vs. shear rate

From data fitting by employing a least-squares method, the equation below was obtained,

$$
\frac{\mathrm{D}_{32}}{\mathrm{~h}}=\mathrm{W} \mathrm{e}^{-0.0045} \mathrm{Re}^{-0.4059}\left(\frac{\mu_{\mathrm{d}}}{\mu_{\mathrm{c}}}\right)^{0.3217}\left(\frac{\rho_{\mathrm{d}}}{\rho_{\mathrm{c}}}\right)^{-6.2485}
$$

Finally, we found that the standard deviation of the droplet size distribution increases linear with the Sauter mean diameter, which yields $\sigma=0.3 \mathrm{D}_{32}$.

\section{CFD simulation}

The main goal is to predict the drop size distribution inside a stirred tank reactor. Thus, efficient and accurate breakup and coalescence models are required in order to simulate the drop size distribution of the liquid-liquid system.

\subsection{Break up model}

The breakup model follows the experimental configuration, where the droplet size distribution can be obtained for a specific shear rate. Thus, from the local shear rate computed from the CFD, the corresponding local equilibrium droplet size distribution due to breakup can be obtained. In particular, fitted correlation for the Sauter mean diameter (equation (5)) is used in each cell (computational domain) inside the stirred tank to produce the local drop size distribution. Finally, actual size of a droplet (which is computed by employing a discrete phase model) is compared to the local equilibrium drop size distribution, which comes from the correlation. If the actual drop is larger than $\mathrm{D}_{90}$, then it is very likely that the drop breaks. It has to be noted that this is actually realized by a random process, where on the one hand the size of the daughter droplets is sampled from the equilibrium distribution and on the other hand a random number is sampled to initialize the breakup itself.

\subsection{CFD Simulation}

To verify the presented approach we compare our numerical simulations with Roudsari et al [10] and Boxall et al [7], who provide detailed simulations and measurements of droplet size distributions in liquidliquid stirred tank reactor. It has to be noted that their system is water-in-oil; however, it is a good verification system, which we can be used as a case study. Roudsari et al [10] used PBE with multiple reference frame method to model the impeller's rotation which was run in the steady state model.

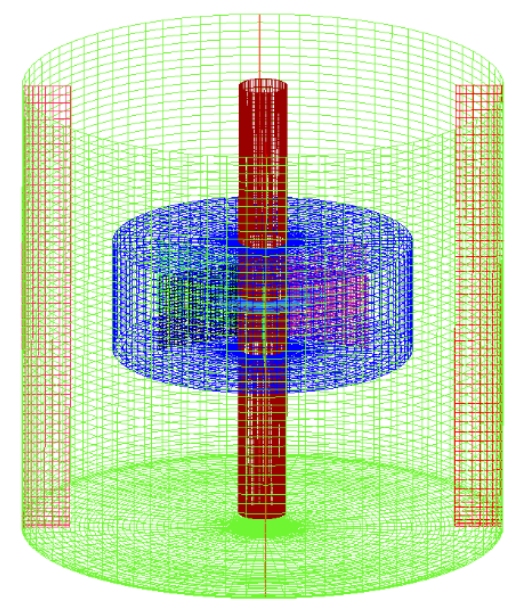

Fig. 4. Stirred tank reactor mesh

Hexahedral mesh of the stirred tank reactor was generated by ANSYS ICEM (Fig.4) and simulations were performed by ANSYS FLUENT 16. A specific oil so-called 'Conrone oil' was used as the continuous phase From Boxall et al [7] and water was selected as the dispersed phase. The Conrone oil density, viscosity and interfacial tensions are $842 \mathrm{~kg} / \mathrm{m}^{3}, 3.1 \mathrm{cP}$ and $20 \mathrm{mN} / \mathrm{m}$, respectively. The volume fraction of water is $15 \%$ in the Boxall et al [7] paper, however in order to avoid the implementation of a model for the coalescence, a linear correction factor (a function of dispersed phase volume fraction) was applied to the Sauter mean diameter (equation (5)), since the correlation was founded for the dispersed phase which has the volume fraction of $1 \%$. Break up model was written as a user-defined function (UDF) to be implemented in ANSYS FLUENT. Eulerian-Lagrangian scheme along with the K- $\varepsilon$ turbulence model was used to simulate the emulsion in the stirred tank reactor. Rotation of the impeller was modelled by sliding mesh method.

\subsection{Results and discussion}

Initial droplets with diameter of $0.3 \mathrm{~mm}$ were injected at $\mathrm{t}=0$. Simulation were performed for $300 \mathrm{rpm}$ and 600 rpm. Simulation stopped when the final drop size distribution did not change any more. Fig.4, 5 show the cumulative drop size distribution for $300 \mathrm{rpm}$ and 600 rpm, respectively. 


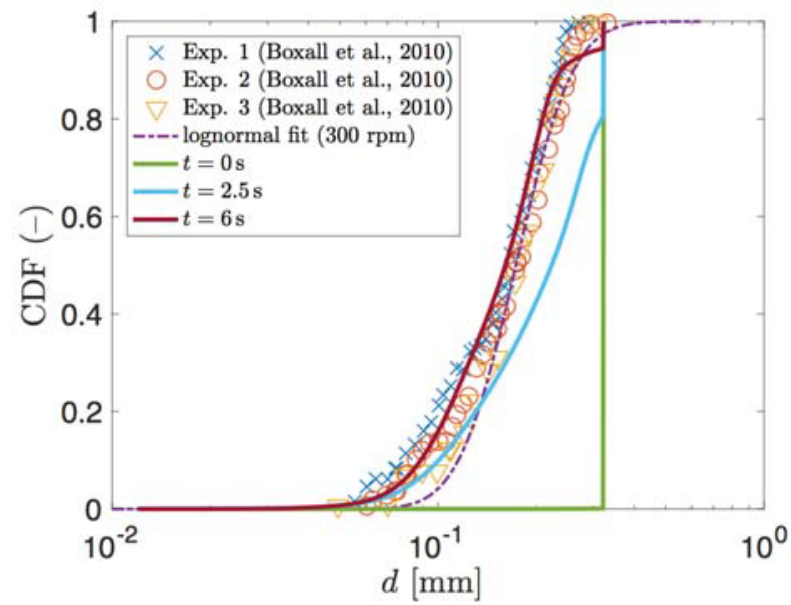

Fig. 5. Cumulative drop size distribution- 15 vol $\%$ water in oil$300 \mathrm{rpm}$

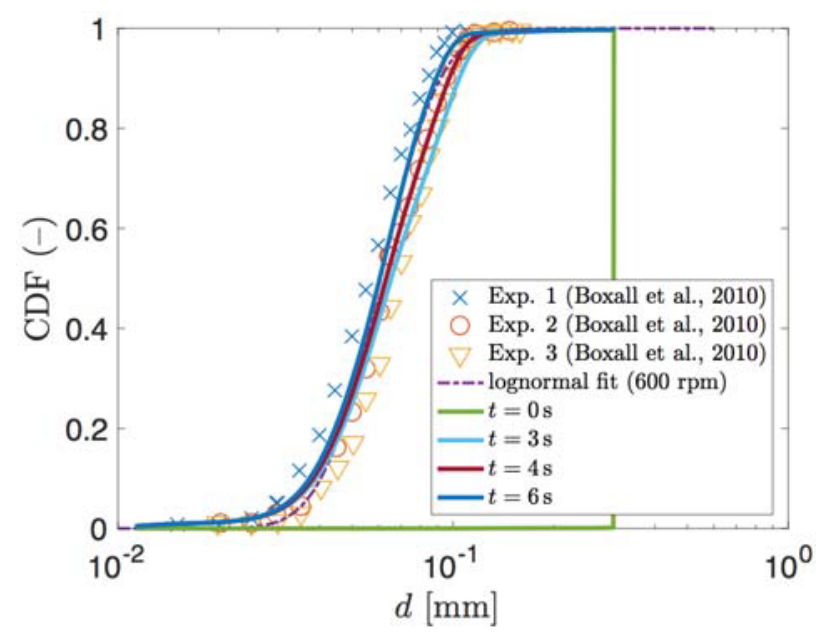

Fig. 6. Cumulative drop size distribution- 15 vol\% water in oil$600 \mathrm{rpm}$

Green line indicates the drop size distribution at $\mathrm{t}=0$. After performing the simulation for 6 seconds in both cases, final results were obtained. Results are in a good agreement with the experimental data from Boxall et al [7]. This model works if the rotational speed goes from the lower speeds to higher one, since there is no coalescence model then simulation is not able to find the correct drop size distribution if the rotational speed is switched from higher speeds to a lower speed.

\section{Conclusion}

Experimental investigation of drop size distribution in a Taylor-Couette device was performed in the current work. Results show that by increasing the Reynolds number, drop size distribution shifts towards the smaller droplets. Also, Sauter mean diameter becomes smaller as shear rate increases. Fitted correlation was used to predict the local drop size distribution in the stirred tank reactor in cooperation with the break up idea. CFD simulation of the liquid-liquid system was done for a specific case and the final results look promising.
Current strategy works only if the rotational speed is going upward and the other way around requires coalescence model.

\section{References}

1. D. E. Leng and R. V. Calabrese, in Handb. Ind. Mix., edited by E. L. Paul, V. A. Atiemo-Obeng, and S. M. Kresta (John Wiley \& Sons, Inc., 2004), pp. 639-753. 2. J. Solsvik and H. A. Jakobsen, Chem. Eng. Sci. 131, 219 (2015).

3. S. Maaß, N. Paul, and M. Kraume, Chem. Eng. Sci. 76, 140 (2012).

4. J. Solsvik, P. J. Becker, N. Sheibat-Othman, I.

Mohallick, R. Farzad, and H. A. Jakobsen, J. Dispers.

Sci. Technol. 36, 577 (2014).

5. D. Ramkrishna, Population Balances (2000).

6. H. T. Chen and S. Middleman, AIChE J. 13, 989 (1967).

7. J. A. Boxall, C. A. Koh, E. D. Sloan, A. K. Sum, and

D. T. Wu, Ind. Eng. Chem. Res. 49, 1412 (2010).

8. R. Shinnar and J. M. Church, Ind. Eng. Chem. 52, 253 (1960).

9. G. Zhou and S. M. Kresta, Chem. Eng. Sci. 53, 2063 (1998).

10. S. F. Roudsari, G. Turcotte, R. Dhib, and F. EinMozaffari, Comput. Chem. Eng. 45, 124 (2012). 